\title{
EFFECTS OF CONTACT LENSES ON SHORT-WAVE FIELD DISTRIBUTION*
}

\author{
BY \\ BRYAN O. SCOTT \\ Department of Physical Medicine, Radcliffe Infirmary, Oxford
}

BOTH THE practice of fitting contact lenses and the application of short-wave therapy to the eye or surrounding tissues are becoming increasingly widespread. It is thus necessary for those concerned with either of these procedures to understand the interrelated effects. It was decided to investigate the effects of contact lenses upon the short-wave field and to elucidate the attendant risk if any.

\section{Method}

Phantom loads were constructed by embedding perspex contact lenses with and without standard perforations at the limbus in a 2 per cent. agar agar gel plate which contained a heat indicator $\left(\mathrm{AgIHgI}_{2}\right)$. Great care was taken with the lenses having perforations to see that the aperture was both filled and level with the surface of the agar plate. A short-wave condenser field was applied through the phantom both antero-posteriorly and laterally in relation to the lens.

\section{Results}

The heating patterns obtained in lenses with perforations are represente in Figs 1 and 2.

In the antero-posterior position the pattern shewed that the current divided in front of the lens and rejoined approximately $2 \mathrm{~cm}$. behind it, suggesting that the lens behaved as a partial screen. In the lenses with a perforation there was heat concentration in and about this (Point P, Fig. 1).

Application of the field laterally appeared to have little effect upon the normal heating pattern, except that there was reduced heat production in the corneal recess (CR, Fig. 2).

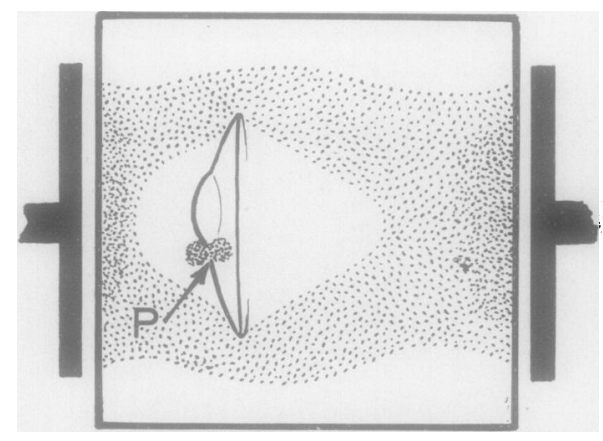

Fig. 1.-Field applied in antero-posterior plane. Heat concentration occurs at perforation $(P)$.
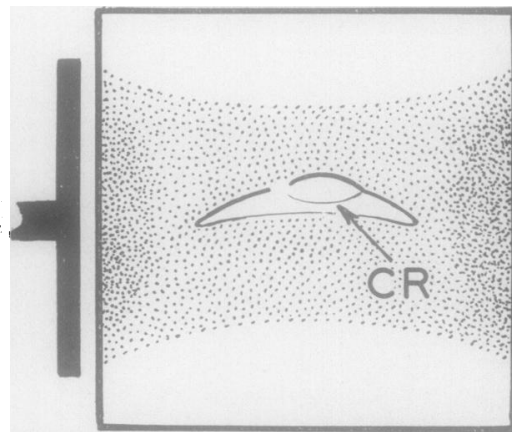

FIG. 2.-Field applied in lateral plane. Little heating occurs in area $\mathbf{C R}$.

* Received for publication July 9, 1956 


\section{Discussion}

These results suggest that, when the contact lens is in situ, the application of condenser field short-wave diathermy to the eye in the antero-posterior plane will result in inefficient heating through screening by the corneal and haptic portions of the lens. Secondly, current concentration at the perforation can occur, with subsequent overheating in the region of the ciliary body and possible serious consequences. Thirdly, even with lateral application, which is an inferior technique, some screening would occur in the anterior chamber.

A corollary, which was suggested by Sarwar (1956) in this connexion, is that the efficiency of short-wave therapy in orbital cellulitis might be increased by fitting a contact lens, without a perforation, during treatment. This would tend to encourage increased current around the eye with more efficient heating of the peri-ocular tissues, but if this effect is not required it appears desirable to remove contact lenses during the application of shortwave therapy to this area.

\section{REFERENCE}

Sarwar, M. (1956). Personal communication

Brit. J. Ophthal. (1956) 40, 697.

\section{METHOD OF CUTTING LAMELLAR GRAFTS* \\ BY \\ P. SIVASUBRAMANIAM \\ Victoria Memorial Eye Hospital, Colombo, Ceylon}

CuTTING a lamellar graft from an intact eyeball is a relatively simple matter, but it is not always possible to secure whole eyes for grafting, and cutting a lamellar graft from an isolated disc of cornea is out of the question unless it is clamped on to a suitable device. Williamson-Noble (1952) suggested sewing the cornea on to the convex end of a finger from a rubber glove stuffed with Dunlopillo rubber.

I have found the following a satisfactory procedure. A vacuum flask cork stopper is shaped to a convex dome with a razor blade and sand-paper. The dome is sterilized by boiling, and the disc of cornea is fixed to it by four small office pins. The cornea is then trephined and the lamella is shaved off.

The advantages of this method are that pinning the cornea on to the cork is easier and quicker than sewing it and the fixation is firmer.

The plastic or bakelite bases of these stoppers, which do not withstand boiling, may be cut off with a knife. The same cork dome may be used again and again to cut penetrating grafts from isolated discs of cornea.

\section{REFERENCE}

Williamson-NoBle, F. A. (1952). British Journal of Ophthalmology, 36, 221.

* Received for publication July 20, 1956. 